\title{
Delirium: A predictor of mortality in the elderly
}

\author{
Matías González MD, PhD* \\ Joan de Pablo MD, $\mathrm{PhD}^{\star \star}$ \\ Manuel Valdés MD, $\mathrm{PhD}^{\star *}$ \\ Silvia Matrai Psychologist ${ }^{\star *}$ \\ Josep Maria Peri Psychologist, PhD** \\ Eduardo Fuente MD**
}

* Departamento de Psiquiatría de la P. Universidad Católica de Chile.

** IDIBAPS, Clinical Institute of Psychiatry and Psychology, Hospital Clínic, University of Barcelona, Spain.

CHILE, SPAIN

\begin{abstract}
The frequency of delirium in elderly inpatients is high, resulting in poor hospital outcomes. The objective of this study is to assess whether delirium is an independent predictor for mortality over a three-month period.

Methods: Prospective, observational study in a cohort of 171 inpatients aged over 65 years. Presence of delirium and/or dementia, severity of delirium and incapacity due to illness were assessed at baseline using DSM-IV diagnostic criteria, the Confusion Assessment Method (CAM), the MMSE, the Delirium Rating Scale (DRS) and the Karnofsky Performance Status (KPS). Mortality rates were evaluated over a three-month follow-up period after enrollment. Kaplan-Meier survival curves were constructed and the adjusted effect of a set of covariates was evaluated with the Cox multiple regression analysis.

Results: By 3 months after enrollment, 34.4\% of the patients with delirium died, compared with $16.5 \%$ of those without delirium. The survival analysis shows a statistically significant difference between the two groups (log-rank=11.92; d.f.=1; $\mathrm{P}=0.0006$ ). After adjustment for covariates, delirium was found to be independently associated with higher mortality.

Conclusions: Delirium was found to be an independent marker for mortality in older medical patients over a three-month follow-up.
\end{abstract}

\section{Introduction}

Delirium is an acute confusional state with global cognitive disfunctioning. The frequency of delirium in the elderly inpatient population ranges from 14 to $56 \%$ (Inouye 1994). The impact of delirium has been studied with relation to its individual and social consequences. The cognitive and functional status after discharge was found to be altered in older inpatients diagnosed with delirium (McCusker et al. 2001), and this condition seems to be an important marker of risk for dementia (Rockwood et al. 1999). The length of stay for different patient groups with delir- 
ium was found to be significantly longer than for non-delirious patients (Ljubisavljevic \& Kelly 2003, Pompei et al. 1994). Poor hospital outcomes such as new nursing home placement and functional decline were also associated with delirium as a prognostic determinant (Inouye et al. 1998). All these facts underline not only the clinical importance of delirium but also its hospital and nursing home costs, which could reach 17.5 million USD per day in the USA (Rizzo et al. 2001).

With respect to mortality for inpatients with delirium, index admission mortality rates were between 9 and 23.9\% (Inouye $e t$ al. 1998, Navinés et al. 2001). Even the prevalent subsyndromal delirium led to a longer hospital stay and increased post discharge mortality in elderly medical inpatients (Cole et al. 2003). Non-detection of delirium in older patients seen in emergency departments may be related to increased mortality after discharge (Kakuma et al. 2003)

Studies assessing the association between delirium and subsequent adverse outcomes including death, reached diverse conclusions. Inouye et al. (1998) reported no significant association between in hospital or threemonth mortality and delirium, while other follow-up studies on post discharge mortality (Rockwood et al. 1999, Francis \& Kapoor 1992) found that delirium could be a marker of risk for death at long term. However, these two studies have limitations such as small sample sizes, limited follow-up and inadequate control of confounding factors. In their 12-month follow-up study, McCusker et al. (2002) introduced a more exhaustive control of these factors and found that delirium was an independent marker for mortality.

Continuing this line of research will contribute to clarify the association between delirium and mortality and increase aware- ness in clinical practice. In this sense, the objective of this study is to report more evidence on the association between mortality and an episode of delirium in the older population over a three-month follow-up. The duration of the follow-up period was established on the basis of previous studies finding that the survival curve for delirious patients decreased rapidly during the first month after enrollment (McCusker et al. 2002).

\section{Methods}

\section{Sample}

The sample was recruited among patients aged over 65 years, admitted over a sixmonth period at the medical and traumatology wards in a 700-bed university-affiliated hospital in Barcelona, Spain, with length of stay longer than 72 hours. Reasons for exclusion were severe aphasia and language barriers. The final sample included 171 inpatients, 65 of whom presented delirium (38.0\%).

\section{Instruments}

A protocol designed for gathering information on clinical features of delirium and dementia was used in the clinical interview. Psychiatric diagnosis for delirium and dementia was determined with the DSM-IV criteria (American Psychiatric Association 1994). The existence of current or previous psychiatric morbidity was established according to data gathered from interviews with the patient, treating physician and relatives, and psychiatric files.

The Confusion Assessment Method (CAM) (Inouye et al. 1990) is a specific index for identifying delirium, based on the operational application of the DSM-III-R 
diagnostic criteria and intended to obtain clinically relevant information from the caregiver (nurse or relative) and the patient. It provides a diagnostic algorithm for delirium based on the presence of the two cardinal features (acute onset and fluctuating course, and inattention) and at least of one of the two secondary features (disorganized thinking and altered level of consciousness). In our study, the Spanish version of the CAM was used (González et al. 2004).

The Delirium Rating Scale (DRS) (Trzepacz et al. 1988) was specifically designed to assess delirium symptoms. It has 10 items and is able to differentiate delirium from other psychiatric conditions. The scores reflect the severity of the symptoms. Both the original and the Spanish versions (Bulbena et al. 1996) used in our study were found to have adequate psychometric properties.

The widely validated Spanish version of the MMSE (Folstein et al. 1975, Lobo et al. 1999) is intended for use by clinicians for the evaluation of cognitive functions such as orientation to time and space, memory, attention and calculation, language and constructional abilities.

The Karnofsky Performance Status (KPS) includes ratings by the clinician of the patient's overall functioning level. Scores of 0 represent minimal functioning while scores of 100 represent unimpaired functioning. It was shown to have good reliability and validity and standardized guidelines for use are also available (Schag et al. 1984). The KPS proved to be highly predictive of outcomes in geriatric patients and to have good ability to discriminate between high- and low-risk groups (Crooks et al. 1991). It also proved to be useful as a predictor of decline in basic daily life activities and quality of life in older patients (Sitjas et al. 2003).

\section{Procedure}

The study was undertaken with the consent of the participants or their legal representative. The patients and their relatives were informed that they would be contacted by telephone in order to evaluate their medical status. A psychiatrist expert in detection of delirium of the Consultation-Liaison (CL) Psychiatry Ward at the Hospital Clínic of Barcelona, selected inpatients with a hospital stay over 72 hours from the medical and orthopedic wards, according to the inclusion criteria. The psychiatrist reviewed the medical record of the patient and interviewed the relatives or the nurses on acute onset and fluctuation of the patient's symptoms of delirium. Then he administered a clinical interview according to DSM-IV criteria and conducted a single cross-sectional evaluation for ratings at the CAM, the MMSE and the KPS. Diagnoses of delirium and dementia were made using all data. If the patient was diagnosed with delirium, the DRS was administered to evaluate the severity of delirium. At three months after enrollment, the $\mathrm{C}$ $\mathrm{L}$ psychiatrist contacted each patient or his/her relative(s) in order to obtain information on vital status. All data were entered twice in a database designed for this purpose.

\section{Statistical Analysis}

Survival curves for patients with and without delirium were constructed with the Kaplan-Meier product limit estimate (Kaplan $\&$ Meier 1958). The significance of differences in survival was tested using the logrank test.

To evaluate the effect of possible prognostic factors in the survival time the Cox multiple regression model was used (Cox \& Oakes 1984). This model identifies and evaluates the relation between a possible set of prog- 
nostic variables and the rate of incidence of the event in question (in our study, death).

$T$ test for independent samples or chisquare statistics were used to compare the group of patients who completed the followup and those lost to follow-up, in order to ensure that there were no significant differences with respect to the main prognostic risk factors.

\section{Results}

Of the 171 patients included in the study, 7 patients were lost to follow-up in the delirium group vs. 15 patients in the non-delirium group, representing $12.9 \%$ of the initial sample (Figure 1). No statistically significant differences were found between these patients and those who completed the study with respect to baseline variables. The rate of delirium in the 149 patients who completed the follow-up was $38.9 \%$ (58 patients). Their characteristics are shown in Table I. The mortality rate at three-month follow-up in the patients with delirium was $34.5 \%$ versus $11 \%$ in those without delirium. When com- paring the mean survival times for patients who died in the delirium group (31.05 days, S.D. 23.16) and for those who died in the non-delirium group (21.8 days, S.D. 11) with the $t$ test for independent samples $(\mathrm{t}=1.18$, $\mathrm{p}=0.24$ ) no statistically significant differences were found.

The Kaplan-Meier survival curve for the delirium cohort declined rapidly over the 50 days after discharge (Figure 2). When comparing the survival curves with the log-rank test, there is a significant difference between survival times in the two cohorts (logrank=11.92; d.f.=1; $\mathrm{P}=0.0006$ ).

We included the following predictive variables in the univariate Cox regression model in order to assess their prognostic effect on mortality: delirium, age, dementia and overall functioning level (KPS). We found that the diagnosis of delirium $\left(\chi^{2}=11.87\right.$; $\mathrm{p}=0.001)$ and the KPS rates $\left(\chi^{2}=4.89\right.$; $\mathrm{p}=0.027$ ) had a significant association with higher mortality. However, in the multivariate model the only remaining explanatory variable was delirium $\left(\chi^{2}=11.29 ; \mathrm{p}=0.001\right)$.

Differences in mean hospital stay were not statistically significant between the two

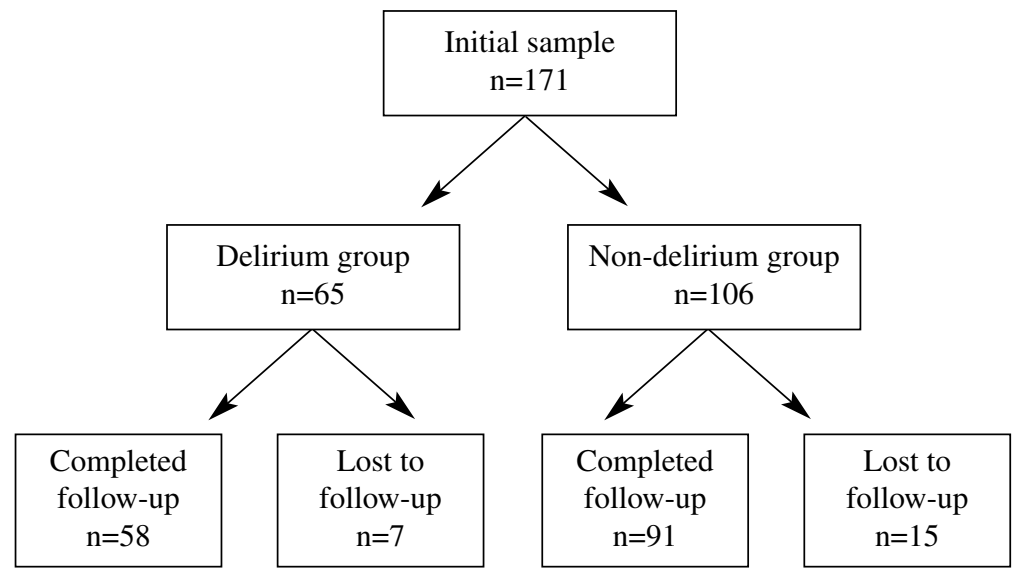

Figure 1. Distribution of the sample over the duration of the study. 
Table I

Comparison of baseline characteristics and rating scale scores between delirious and non-delirious subjects who completed the follow-up

\begin{tabular}{|c|c|c|c|c|}
\hline & \multicolumn{2}{|c|}{ Delirium Cohort $(n=65)$} & \multicolumn{2}{|c|}{ Non-delirium Cohort $(n=106$} \\
\hline & $\begin{array}{l}\text { completed } \\
\text { follow-up }\end{array}$ & $\begin{array}{c}\text { lost to } \\
\text { follow-up }\end{array}$ & $\begin{array}{l}\text { completed } \\
\text { follow-up }\end{array}$ & $\begin{array}{c}\text { lost to } \\
\text { follow-up }\end{array}$ \\
\hline $\mathrm{N}$ & 58 & $7(10.8 \%)$ & 91 & $15(14.1 \%)$ \\
\hline Mortality $(n)$ & $20(34.5 \%)$ & & $10(11.0 \%)$ & \\
\hline \multicolumn{5}{|l|}{ Gender $(n)$} \\
\hline Male & $25(43.1 \%)$ & $2(28.6 \%)$ & $40(44.0 \%)$ & $4(26.7 \%)$ \\
\hline Female & $33(56.9 \%)$ & $5(71.4 \%)$ & $51(56.0 \%)$ & $11(73.3 \%)$ \\
\hline \multicolumn{5}{|l|}{ Primary medical diagnosis $(n)$} \\
\hline Cardiorespiratory & $12(20.7 \%)$ & $1(14.3 \%)$ & $23(25.3 \%)$ & $4(26.7 \%)$ \\
\hline Metabolic & $7(12.1 \%)$ & $2(28.6 \%)$ & $13(14.3 \%)$ & $2(13.3 \%)$ \\
\hline Infectious & $2(3.4 \%)$ & $1(14.3 \%)$ & $3(3.3 \%)$ & $0(0.0 \%)$ \\
\hline Orthopedic & $22(37.9 \%)$ & $2(28.6 \%)$ & $39(42.8 \%)$ & $5(33.3 \%)$ \\
\hline Others & $15(25.9 \%)$ & $1(14.3 \%)$ & $13(14.3 \%)$ & $4(26.7 \%)$ \\
\hline Dementia $(n)$ & $30(51.7 \%)$ & $4(57.1 \%)$ & $41(45.0 \%)$ & $10(66.7 \%)$ \\
\hline Mean age (years) (SD) & $78.47(6.68)$ & $82.14(7.34)$ & $76.36(7.40)$ & $76.93(6.81)$ \\
\hline Mean hospital stay (SD) (days) & $22.62(21.37)$ & $21.83(21.19)$ & $18.67(19.96)$ & $19.74(20.02)$ \\
\hline Mean (SD) KPS 0-100 & $13.97(9.54)$ & $12.86(4.88)$ & $26.15(12.45)$ & $25.33(13.56)$ \\
\hline Mean (SD) DRS rating & $21.55(5.86)$ & $20.57(5.88)$ & not adm & inistered \\
\hline Mean (SD) MMSE rating & $13.05(8.49)$ & $15.14(7.10)$ & $23.90(6.78)$ & $21.07(6.49)$ \\
\hline
\end{tabular}

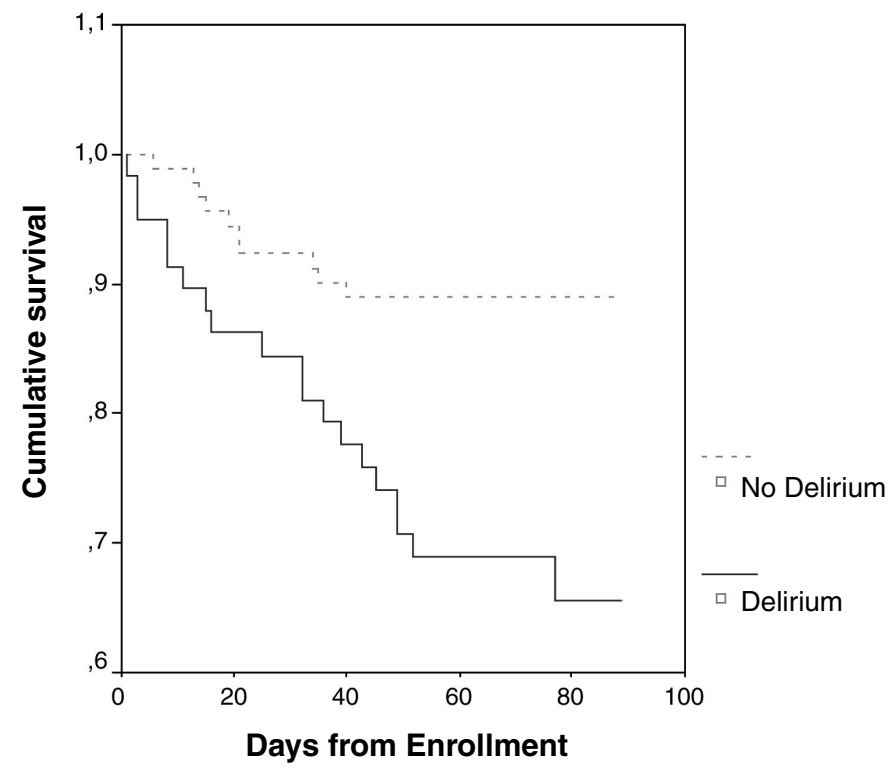

Figure 2. Kaplan-Meier 3-month survival curves by delirium and non-delirium cohorts. 
groups: 22.62 days $(\mathrm{SD}=21.37)$ in the delirium vs. 18.67 days $(\mathrm{SD}=19.96)$ in the nondelirium group $(t=1,129 ; \mathrm{p}=0.261)$.

\section{Discussion}

The results of this study confirm the association of delirium with a higher risk for mortality and are in accordance with the findings of previous studies (Rockwood et al. 1999, Francis \& Kapoor 1992). Moreover, delirium was showed to be an independent marker for increased mortality, in line with the conclusion of McCusker et al. (2002). However, this result has not been confirmed in all studies (Inouye et al. 1998) This could be due to the fact that the objective of the cited study was to assess hospital outcomes in patients with delirium, and therefore a large number of variables were assessed which can be confounding in the evaluation of mortality as an independent factor.

The selection of the prognostic factors included in our model was based on the results of previous studies showing their impact on the mortality rates. The functional state (KPS) and delirium were associated with the risk of death in the univariate regression model. However, after adjusting for all the assessed factors including dementia and age, we found that only delirium remained independently associated with mortality over the follow-up period.

There are no statistically significant differences in mean survival time between the two groups (delirium and no delirium), and the functional state (KPS) has no independent association with mortality in the final model. Both of these findings could be explained by the severe decline in the functioning level (mean KPS=21.41, S.D.=12.84) at baseline in the study population, which contributes to shorten the survival time in both groups and makes more difficult the comparison between them with respect to global functioning level.

In contrast to the study of McCusker et al. (2002), the variable 'age' was not found to be independently associated with higher mortality in our study. We consider that the reduced follow-up period in our sample could prevent a significant impact of the factor 'age'.

It is important to highlight that our followup period ( 3 months) is much shorter than in the cited studies (3 years and 12 months, respectively) (Rockwood et al. 1999, McCusker et al. 2002). The survival curves of previous studies show an important decline during the first month followed by a deceleration. Therefore, we assessed a shorter follow-up period, as the effect of delirium on mortality may decrease while the effect of confounding factors may increase with time, which could bias the results over the long run even after statistical adjustment.

The fact that differences in mean hospital stay did not reach statistical differences between the delirium and non-delirium groups in our study, in contrast to other studies, could be explained by the similar grade of impairment of the functional level associated with physical illness in both groups.

Our study has some limitations. The study sample was selected from hospital wards with a high percentage of elderly patients at admission and therefore more severe morbidity due to age. Another limitation might be due to the relatively small sample size and the limited follow-up period. Further studies in different hospital settings should confirm our results in larger samples with longer follow-up periods. Moreover, the presence of delirium was assessed at 72 hours of hospitalization with no information on the existence of previous or subsequent delirious episodes, which could contribute false nega- 
tive cases to appear in the delirium group. Finally, the functional state assessed with the KPS was used as an indirect measure of severity, while higher accuracy could have been reached by using more specific indicators of the severity of physical illness.

In sum, the results of this study are in line with previous studies confirming the association between delirium and mortality, and point out the effect of delirium on the prognosis over a short period after the acute episode.

\section{References}

American Psychiatric Association. Diagnostic and statistical manual of mental disorders (DSM-IV), 4th ed. Washington D.C.: APA; 1994.

Bulbena A, Corrons C, Amorós G et al. Escalas de delirium y orientación. Adaptación castellana del Delirium Rating Scale y de la Orientation Scale. Rev Gerontol 1996; 6: 245254.

Cole M, Mccusker J, Dendukuri N et al. The prognostic significance of subsyndromal delirium in elderly medical inpatients. J Am Geriatr Soc 2003; 51(6): 754-60.

Cox Dr, Oakes D. Analysis of survival data. London: Chapman \& Hall; 1984.

Crooks V, Waller S, Smith T et al. The use of the Karnofsky Performance Scale in determining outcomes and risk in geriatric outpatients. J Gerontol 1991 ; 46: M139-M144.

Folstein MF, Folstein SE, Mchugh PR. "Mini-Mental State": A practical method for grading the cognitive state of patients for the clinician. J Psychiatr Res 1975; 12: 189-198.

Francis J, Kapoor WN. Prognosis after hospital discharge of older medical patients with delirium. Jags 1992; 40: 601606.

Inouye SK, Rushing JT, Foreman MD et al. Does delirium contribute to poor hospital outcomes?. A three-site epidemiologic study. J Gen Intern Med 1998; 13: 234-242.

Inouye SK, Van Dyck CH, Alessi CA et al. Clarifying confusion: the confusion assessment method. A new method for detection of delirium. Ann Inter Med 1990; 113: 941-948.

Inouye SK. The dilemma of delirium: Clinical and research controversies regarding diagnosis and evaluation of delirium in hospitalized elderly medical patients. Am J Medicine 1994 ; 97: 278-288.
Kakuma R, Du Fort Gg, Arsenault L et al. Delirium in older emergency department patients discharged home: Effect on survival. J Am Geriatr Soc 2003; 51(4): 443-50.

Kaplan EL, Meier P. Non-parametric estimations from incomplete observations. J Am Statistical Assoc 1958; 53: 457-481.

Ljubisavljevic V, Kelly B. Risk factors for development of delirium among oncology patients. Gen Hosp Psychiatry 2003; 25(5): 345-52.

Lobo A, Saz P, Marcos G et al. Revalidation and standardization of the Mini-Exam (First spanish version of the Mini-Mental Status Examination) in the general geriatric population. Med Clin (Barc) 1999; 112(20): 767-774

Mccusker J, Cole M, Abrahamowicz M et al. Delirium predicts 12-month mortality. Arch Intern Med 2002; 162: 457-463.

Mccusker J, Cole M, Dendukuri N et al. Delirium in older medical inpatients and subsequent cognitive and functional status: A prospective study. Cmaj 2001; 165(5): 575-83.

Navinés R, Gómez E, Franco JG et al. Delirium in a consultation-liaison psychiatry unit of a general hospital. Actas Esp Psiquiatr 2001; 29(3): 159-164.

Pompei P, Foreman M, Rudberg MA et al. Delirium in hospitalized older persons: Outcomes and predictors. $J$ Am Geriatr Soc 1994; 42(8): 809-15.

Rizzo Ja, Bogardous St Jr, Leo-Summers Let al. Multicomponent targeted intervention to prevent delirium in hospitalized older patients: What is the economic value?. Med Care 2001; 39(7): 740-752.

Rockwood K, Cosway S, Carver D et al. The risk of dementia and death after delirium. Age Ageing 1999; 28: 551-556.

Schag CC, Heinrich RL, Ganz PA. Karnofsky Performance Status Revisited: Reliability validity and guidelines. J Clin Oncology 1984; 2: 187-193.

Sitjas E, San Jose A, Armadans L et al. Predictor factors about functional decline in community-dwelling older persons. Aten Primaria 2003; 32(5): 282-287.

Trzepacz PT, Baker RW, Greenhouse J. A Symptom Rating Scale for delirium. Psychiatry Res 1988; 23: 89-97.

Address for correspondence:

Joan de Pablo

Sección de Psiquiatría de Enlace del Instituto Clínico de Psiquiatría y Psicología

Hospital Clínico de Barcelona

Villaroel, 170

08036 Barcelona

jdepablo@clinic.ub.es

SPAIN 